\title{
THE LINEAR SPACE OF BETTI DIAGRAMS OF MULTIGRADED ARTINIAN MODULES
}

\author{
GunNAR FløYSTAD
}

\begin{abstract}
We study the linear space generated by the multigraded Betti diagrams of $\mathbb{Z}^{n}$-graded artinian modules of codimension $n$ whose resolutions become pure of a given type when taking total degrees. We show that the multigraded Betti diagram of the equivariant resolution constructed in [3] by D.Eisenbud, J.Weyman, and the author, and all its twists, form a basis for this linear space. We also show that it is essentially unique with this property.
\end{abstract}

\section{Introduction}

Recent years has seen a breakthrough in the studies of syzygies of graded modules over the polynomial ring $S=\mathbb{k}\left[x_{1}, \ldots, x_{n}\right]$. In [1], M.Boij and J.Söderberg formulated conjectures describing the positive cone of Betti diagrams of artinian modules over the polynomial ring. The conjectures were subsequently proven by the work of D.Eisenbud, J.Weyman, and the author in [3], and by Eisenbud and F.-O. Schreyer in [4]. Fundamental in showing the conjectures is to show the existence of pure resolution of artinian modules. A resolution is pure if it has the form

$$
S\left(-d_{0}\right)^{\beta_{0}} \leftarrow S\left(-d_{1}\right)^{\beta_{1}} \leftarrow \cdots \leftarrow S\left(-d_{n}\right)^{\beta_{n}}
$$

for a sequence $\mathbf{d}: d_{0}<d_{1}<\cdots<d_{n}$. In [3] the existence of such resolutions of graded artinian modules is shown for every sequence $\mathbf{d}$ when $\operatorname{char} \mathbb{k}=0$. Moreover the construction there is a quite explicit $G L(n)$-equivariant resolution. In particular it is equivariant for the diagonal matrices and hence $\mathbb{Z}^{n}$-graded.

The beauty and naturality of this resolution is apparent from the construction. It has recently been generalised by S.Sam and Weyman in [8] to wider classes of equivariant resolutions. In this paper we consider the class of resolutions of $\mathbb{Z}^{n}$ graded artinian modules which become pure when taking total degrees. We establish, in a precise sense, that the equivariant resolution constructed in [3], or rather its multigraded Betti diagram, is the fundamental resolution in this class.

Before stating the results more precisely, let us consider a simple example for illustration. If $F_{\bullet}$ is a resolution of a $\mathbb{Z}^{n}$-graded module, we have a twisted complex $F_{\bullet}$ (a) for $\mathbf{a} \in \mathbb{Z}^{n}$. If $\beta$ is the Betti diagram of $F_{\bullet}$, then $F_{\bullet}$ (a) will have a Betti diagram which we denote by $\beta(\mathbf{a})$.

Example 0.1. The following example was worked out together with J.Weyman. Let $S=\mathbb{k}\left[x_{1}, x_{2}\right]$ and suppose $d_{1}-d_{0}=2$ and $d_{2}-d_{1}=3$. The equivariant resolution

Received by the editors March 26, 2010.

2000 Mathematics Subject Classification. Primary: 13D02; Secondary: 13C14.

Key words and phrases. keywords. 
has the following form where we have written the bidegrees of the generators below the terms.

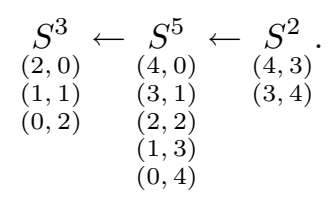

Let $\beta_{1}$ be its bigraded Betti table. In [1, Remark 3.2] Boij and Söderberg also gave a construction of pure resolutions in the case of two variables. These were resolutions of a quotient of a pair of monomial ideals. For the type above the resolution had the following bidegrees.

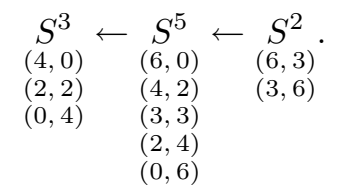

Let $\beta_{2}$ be its Betti diagram. Then $\beta_{2}$ is a linear combination of twists of $\beta_{1}$ but not vice versa: $\beta_{2}=\beta_{1}(2,0)-\beta_{1}(1,1)+\beta_{1}(0,2)$. This indicates that in some way the complex (1), or at least its Betti diagram, is more fundamental than that of the complex (2).

Now let $e_{i}=d_{i}-d_{i-1}$ and let $\mathbf{e}$ be the sequence of these differences. Consider $\mathbb{Z}^{n}$ graded resolutions and their Betti diagrams, of artinian $\mathbb{Z}^{n}$-graded modules over the polynomial ring $S$ of dimension $n$. We shall be interested in those resolutions which become pure when taking total degrees and for which the difference sequence of these total degrees is e. Let $L(\mathbf{e})$ be the $\mathbb{Q}$-vector space generated by such Betti diagrams. Our result is a complete description of this vector space when $\mathbb{k}$ has characteristic 0 . For the given e, consider the equivariant resolution constructed in [3], which has $\mathbf{e}$ as difference vector of the total degrees, and let $\beta$ (which of course depends on $\mathbf{e}$ ) be its $\mathbb{Z}$-graded Betti diagram. Also let $r$ be the greatest common divisor of $e_{1}, \ldots, e_{n}$. In the case $r=1$ our main result Theorem 1.4 says the following.

Theorem. The set of twisted diagrams $\beta(\mathbf{a})$ where $\mathbf{a} \in \mathbb{Z}^{n}$, constitute a basis for the lattice of integral points in $L(\mathbf{e})$. Moreover, up to a twist $\mathbf{a}, \beta$ is the unique Betti diagram with this property.

In particular the $\beta(\mathbf{a})$ where $\mathbf{a} \in \mathbb{Z}^{n}$ form a basis for the vector space $L(\mathbf{e})$.

This theorem shows the canonical stature of the multigraded Betti diagram of the equivariant resolution.

For arbitrary $r$ the theorem holds true but with $\beta$ being the Betti diagram of a somewhat modified resolution. We consider the equivariant resolution with difference sequence $\mathbf{e}^{\prime}$ where $\mathbf{e}=r \cdot \mathbf{e}^{\prime}$. By replacing $x_{i}$ by $x_{i}^{r}$ in this resolution corresponding to $\mathbf{e}^{\prime}$, we obtain a resolution with difference sequence $\mathbf{e}$, and now let $\beta$ be the Betti diagram of this complex. With this modification the above theorem holds for any $r$ (when $\mathbb{k}$ has characteristic 0 ).

The Betti diagrams of $\mathbb{Z}^{n}$-graded artinian modules fulfil a multigraded version of the Herzog-Kühl equations. We then introduce the $\mathbb{Q}$-vector space $L^{\prime}(\mathbf{e})$ generated by $\mathbb{Z}^{n}$-graded diagrams (that need not arise from resolutions) whose total degrees are 
pure with difference vector $\mathbf{e}$, and which fulfil the multigraded Herzog-Kühl equations. Note that the field $\mathbb{k}$ is not involved in the definition of this linear space.

There is a natural injection $L(\mathbf{e}) \rightarrow L^{\prime}(\mathbf{e})$. With $L(\mathbf{e})$ replaced by $L^{\prime}(\mathbf{e})$, the theorem above also holds and is the essential part of our first main Theorem 1.2 (this does not involve $\mathbb{k}$ ). This statement will imply that $L^{\prime}(\mathbf{e})$ may be identified with the Laurent polynomial ring $\mathbb{Q}\left\{t_{1}, \ldots, t_{n}\right\}$, and $L(\mathbf{e})$ may be identified as an ideal $\mathscr{I}(\mathbb{k} ; \mathbf{e})$ in this ring. When $\mathbb{k}$ has characteristic 0 , what we say above shows that this ideal is the whole ring. But when it has characteristic $p$ it is an intriguing question, which we do not know much about, to describe this ideal.

In the case where the resolutions are simply $\mathbb{Z}$-graded instead of $\mathbb{Z}^{n}$-graded, one may also consider the vector space $L(\mathbf{e})$. This decomposes as a sum of one-dimensional spaces $L(\mathbf{d})$, one for each $\mathbf{d}$ with difference sequence equal to $\mathbf{e}$. Taking the lattice of integer points in $L(\mathbf{d})$, it is conjectured in [3] that all sufficiently large diagrams may be realised as Betti diagrams of resolutions. There are however positive diagrams which are not realised by any resolution, see also [5] and [2] for more in this direction. Our result implies, see Corollary 1.6, that in the $\mathbb{Z}^{n}$-graded case, if you take an integer lattice point of $L(\mathbf{d})$, a $\mathbb{Z}^{n}$-graded diagram, and form the $\mathbb{Z}$-graded diagram from it by taking total degrees, then such a diagram must be an integer multiple of the $\mathbb{Z}$-graded Betti diagram associated to the equivariant resolution.

Of course, even more interesting than the linear space $L(\mathbf{e})$ is the positive rational cone $P(\mathbf{e})$ generated by the Betti diagrams of resolutions of $\mathbb{Z}^{n}$-graded artinian modules which are pure with respect to total degrees and with difference sequence e of the total degrees. It is considerably more difficult to describe this cone. In the paper [7] by Boij and the author, we describe this cone completely in the case of two variables, and give some examples in the case of three variables.

The organisation of the paper is as follows. In Section 1 we give basic facts and notations. We note that multigraded Betti diagrams fulfil strong numerical criteria, the multigraded versions of the Herzog-Kühl equations. We recall the form of the equivariant complex, and state our main results concerning the basis of the linear space $L^{\prime}(\mathbf{e})$, and that $L(\mathbf{e})$ identifies with this space when $\mathbb{k}$ has characteristic 0 . The terms of the equivariant resolution are of the form $S \otimes_{k} S_{\lambda}$ for a Schur module $S_{\lambda}$. In Section 2 we study the associated Schur polynomials $s_{\lambda}$ of the terms in the resolution. In order to establish our main result, we find the greatest common divisor of these polynomials. In Section 3 we describe the structure of the linear space $L^{\prime}(\mathbf{e})$. From this description and the results in Section 2 concerning Schur polynomials, we give the (immediate) proofs of the main theorems describing $L(\mathbf{e})$ and $L^{\prime}(\mathbf{e})$.

\section{The linear space of multigraded Betti diagrams}

In this section we give the basic facts and notations concerning multigraded Betti diagrams. We describe the multigraded Herzog-Kühl equations. We recall the construction of pure resolutions in [3]. In the end we give the statement of our main result.

1.1. Betti diagrams and the Herzog-Kühl equations. Let $S=\mathbb{k}\left[x_{1}, \ldots, x_{n}\right]$ be the polynomial ring over a field $\mathbb{k}$. We shall study $\mathbb{Z}^{n}$-graded free resolutions of 
$\operatorname{artinian} \mathbb{Z}^{n}$-graded $S$-modules

$$
F_{0} \leftarrow F_{1} \leftarrow \cdots \leftarrow F_{n}
$$

For a multidegree $\mathbf{a}=\left(a_{1}, a_{2}, \ldots, a_{n}\right)$ in $\mathbb{Z}^{n}$ let $|\mathbf{a}|=\sum a_{i}$ be its total degree. We shall be interested in the case that these resolutions become pure if we make them singly graded by taking total degrees. So there is a sequence $d_{0}<d_{1}<\cdots<d_{n}$ such that

$$
F_{i}=\oplus_{|\mathbf{a}|=d_{i}} S(-\mathbf{a})^{\beta_{i, \mathbf{a}}} .
$$

The multigraded Betti diagram of such a resolution is the element

$$
\left\{\beta_{i, \mathbf{a}}\right\}_{i=\underset{\mathbf{a} \in \mathbb{Z}^{n}}{0, \ldots, n}} \in \oplus_{\mathbb{Z}^{n}} \mathbb{N}^{n+1} .
$$

A way of representing a multigraded Betti diagram which will turn out very convenient for us, is to represent $\beta=\left\{\beta_{i, \mathbf{a}}\right\}$ where $i=0, \ldots, n$ and $\mathbf{a} \in \mathbb{Z}^{n}$ by Laurent polynomials

$$
B_{i}(t)=\sum_{\mathbf{a} \in \mathbb{Z}^{n}} \beta_{i, \mathbf{a}} \cdot t^{\mathbf{a}} .
$$

We call this the Betti polynomial of the diagram $\beta$ or the resolution $F_{\bullet}$. We thus get an $(n+1)$-tuple of Laurent polynomials

$$
B=\left(B_{0}, B_{1}, \ldots, B_{n}\right) .
$$

Given a set of total degrees $\mathbf{d}: d_{0}<d_{1}<\cdots<d_{n}$. Let $L(\mathbf{d})$ in $\oplus_{\mathbb{Z}^{n}} \mathbb{Q}^{n+1}$ be the linear subspace generated by multigraded Betti diagrams of artinian $\mathbb{Z}^{n}$-graded modules whose resolutions become pure of degrees $d_{0}, \ldots, d_{n}$ after taking total degrees.

Furthermore let $e_{i}=d_{i}-d_{i-1}$. This gives the difference vector $\Delta \mathbf{d}=\mathbf{e}=$ $\left(e_{1}, \ldots, e_{n}\right)$. Most of the time it will be convenient to fix the difference vector instead of the vector of total degrees. We therefore let $L(\mathbf{e})=\oplus_{\Delta \mathbf{d}=\mathbf{e}} L(\mathbf{d})$ be the linear subspace of $\oplus_{\mathbb{Z}^{n}} \mathbb{Q}^{n+1}$ generated by all multigraded Betti diagrams which become pure when considering total degrees, and where the difference vector of these total degrees is $\mathbf{e}$.

There are some natural restrictions on $L(\mathbf{e})$ coming from the multigraded HerzogKühl equations, drawn to my attention by M.Boij. If the resolution resolves the module $M$, the multigraded Hilbert series of $M$ is

$$
h_{M}(t)=\frac{\sum_{i, \mathbf{a}}(-1)^{i} \beta_{i, \mathbf{a}} \cdot t^{\mathbf{a}}}{\Pi_{k=1}^{n}\left(1-t_{i}\right)} .
$$

If $M$ is artinian, $h_{M}(t)$ is a polynomial and

$$
\sum_{i, \mathbf{a}}(-1)^{i} \beta_{i, \mathbf{a}} t^{\mathbf{a}}=h_{M}(t) \cdot \Pi_{k=1}^{n}\left(1-t_{i}\right) .
$$

For each multigraded $\mathbf{a} \in \mathbb{Z}^{n}$ and integer $k=1, \ldots, n$, let the projection $\pi_{k}(\mathbf{a})$ be $\left(a_{1}, \ldots, \hat{a}_{k}, \ldots, a_{n}\right)$, the $(n-1)$-tuple where we omit $a_{k}$.

We obtain the multigraded analogs of the Herzog-Kühl (HK) equations by setting $t_{k}=1$ in (3) for each $k$. This gives for every $\hat{\mathbf{a}}$ in $\mathbb{Z}^{n-1}$ and $k=1, \ldots, n$ an equation

$$
\sum_{i, \pi_{k}(\mathbf{a})=\hat{\mathbf{a}}}(-1)^{i} \beta_{i, \mathbf{a}}=0 .
$$


Now let $L^{\prime}(\mathbf{e})$ be the linear space of elements in $\oplus \mathbf{a} \in \mathbb{Z}^{n} \mathbb{Q}^{n+1}$ which fulfil the multigraded HK-equations above, and become pure diagrams when taking total degrees, and with the difference sequence of these total degrees equal to e. There is a natural injection $L(\mathbf{e}) \rightarrow L^{\prime}(\mathbf{e})$. Note that $L^{\prime}(\mathbf{e})$ does not depend on the field $\mathbb{k}$, but $L(\mathbf{e})$ does. Our second main Theorem 1.4 states that this map is an isomorphism in characteristic 0 .

1.2. The equivariant resolution and Schur polynomials. In [3] the author together with D.Eisenbud and J.Weyman constructed a $G L(n)$-equivariant pure resolution of an artinian module, whose form we now describe. For a partition $\lambda=$ $\left(\lambda_{1}, \ldots, \lambda_{n}\right)$ let $S_{\lambda}$ be the associated Schur module, which is an irreducible representation of $G L(n)$ (see for instance [6]). The action of the diagonal matrices in $G L(n)$ gives a decomposition of $S_{\lambda}$ as a $\mathbb{Z}^{n}$-graded vector space. The basis elements are given by semi-standard Young tableau of shape $\lambda$ with entries from $1,2, \ldots, n$. All the nonzero graded pieces in this decomposition have total degree $|\lambda|=\sum_{i=1}^{n} \lambda_{i}$. The free module $S \otimes_{k} S_{\lambda}$ then becomes a free multigraded module where the generators all have total degree $|\lambda|$.

Now given the difference vector $\mathbf{e}$, let

$$
\lambda_{i}=\sum_{j=i+1}^{n}\left(e_{j}-1\right)
$$

and define a sequence of partitions for $i=0, \ldots, n$ by

$$
\alpha(\mathbf{e}, i)=\left(\lambda_{1}+e_{1}, \lambda_{2}+e_{2}, \ldots, \lambda_{i}+e_{i}, \lambda_{i+1}, \ldots, \lambda_{n}\right) .
$$

The construction in [3] then gives a $G L(n)$-equivariant resolution

$$
E(\mathbf{e}): S \otimes_{k} S_{\alpha(\mathbf{e}, 0)} \leftarrow S \otimes_{k} S_{\alpha(\mathbf{e}, 1)} \leftarrow \cdots \leftarrow S \otimes_{k} S_{\alpha(\mathbf{e}, n)}
$$

of an artinian $S$-module. Note that our notation differs somewhat from [3]. There the $\alpha$ 's depend on $\mathbf{d}$ while we use the difference vector as argument.

The Betti polynomial of $S \otimes_{k} S_{\lambda}$ will be the character of $S_{\lambda}$ which is the Schur polynomial $s_{\lambda}$. For a matrix $\left(a_{i j}\right)$ where $i, j=1, \ldots, n$, let $\left|a_{i j}\right|$ denote the determinant of the matrix. The Schur polynomial is then given by the expression

$$
s_{\lambda}=\frac{\left|t_{i}^{\lambda_{j}+n-j}\right|}{\left|t_{i}^{n-j}\right|} .
$$

Note that the denominator here is $D=\Pi_{i<j}\left(t_{j}-t_{i}\right)$.

It is also interesting to note the following.

Lemma 1.1. For $i=0, \ldots, n$, the $i$ 'th Betti polynomial $B_{i}$ associated to the equivariant complex $E(\mathbf{e})$, is the maximal minor obtained by deleting column $n-i$ in the $n \times(n+1)$ matrix

$$
\left[\begin{array}{ccccc}
1 & t_{1}^{e_{n}} & t_{1}^{e_{n}+e_{n-1}} & \cdots & t_{1}^{e_{n}+e_{n-1}+\cdots+e_{1}} \\
1 & t_{2}^{e_{n}} & t_{2}^{e_{n}+e_{n-1}} & \cdots & t_{2}^{e_{n}+e_{n-1}+\cdots+e_{1}} \\
\vdots & & & & \\
1 & t_{n}^{e_{n}} & t_{1}^{e_{n}+e_{n-1}} & \cdots & t_{n}^{e_{n}+e_{n-1}+\cdots+e_{1}}
\end{array}\right]
$$

divided by $D=\Pi_{i<j}\left(t_{j}-t_{i}\right)$. 
Proof. Let $\rho=(n-1, n-2, \cdots, 1,0)$. The partition $\alpha(\mathbf{e}, i)$ is then

$$
\left(\sum_{1}^{n} e_{j}, \sum_{2}^{n} e_{j}, \ldots, \sum_{i}^{n} e_{j}, \sum_{i+2}^{n} e_{j}, \ldots, 0\right)-\rho
$$

and the associated Schur polynomial is then the minor we get in the matrix above by omitting column $n-i$, and dividing by $D$.

1.3. The linear space $L(\mathbf{e})$. For a multigraded Betti diagram $\beta=\left\{\beta_{i, \mathbf{a}}\right\}$ and a multidegree $\mathbf{t}$ in $\mathbb{Z}^{n}$, we get the twisted Betti diagram $\beta(-\mathbf{t})$ which in homological degree $i$ and multidegree $\mathbf{a}$ is given by $\beta_{i, \mathbf{a}-\mathbf{t}}$. If $F$. is a resolution with Betti diagram $\beta$, then $F$. $(-\mathbf{t})$ is a resolution with Betti diagram $\beta(-\mathbf{t})$.

Also let $F_{r}: S \rightarrow S$ be the map sending $x_{i} \mapsto x_{i}^{r}$. Denote by $S^{(r)}$ the ring $S$ with the $S$-module structure given by $F_{r}$. Given any complex $F$. we may tensor it with $-\otimes_{S} S^{(r)}$ and get a complex we denote by $F^{(r)}$. Note that if $F$. is pure with degrees $\mathbf{d}$, then $F^{(r)}$ is pure with degrees $r \cdot \mathbf{d}$.

The following are our main results and shows that the numerical part of the equivariant complex, its multigraded Betti diagram, plays the fundamental role when considering multigraded Betti diagrams of resolutions of artinian $\mathbb{Z}^{n}$-graded modules.

Theorem 1.2. Let $r=\operatorname{gcd}\left(e_{1}, \ldots, e_{n}\right)$ and let $\mathbf{e}=r \cdot \mathbf{e}^{\prime}$. The Betti diagrams $\beta_{E\left(\mathbf{e}^{\prime}\right)^{(r)}}(\mathbf{a})$ where a varies over $\mathbb{Z}^{n}$, form a basis for the lattice of integral points in $L^{\prime}(\mathbf{e})$. Moreover $\beta_{E\left(\mathbf{e}^{\prime}\right)^{(r)}}$ is, up to sign and twist with $\mathbf{a} \in \mathbb{Z}^{n}$, the unique element in $L^{\prime}(\mathbf{e})$ with this property.

In particular, the $\beta_{E\left(\mathbf{e}^{\prime}\right)^{(r)}}(\mathbf{a})$ where $\mathbf{a}$ varies over $\mathbb{Z}^{n}$ form basis for the vector space $L^{\prime}(\mathbf{e})$.

The proof will be given in Section 3. The first part of Theorem 1.2 may also be formulated in an equivalent way in terms of the associated $(n+1)$-tuple of Betti polynomials introduced at the end of Subsection 1.1 .

Theorem 1.2' Let $s=\left(s_{0}, \ldots, s_{n}\right)$ be the $(n+1)$-tuple of Betti polynomials of $E\left(\mathbf{e}^{\prime}\right)^{(r)}$. If $B=\left(B_{0}, \ldots, B_{n}\right)$ is any $(n+1)$-tuple of homogeneous Laurent polynomials fulfilling the HK-equations (4), and where the difference vector of the total degrees is $\mathbf{e}$, then $B=p \cdot s$ for some homogeneous Laurent polynomial $p$.

Example 1.3. Letting $B_{(1)}$ and $B_{(2)}$ be the triples of Betti polynomials of the resolutions (1) and (2) of the example in the introduction, we have

$$
B_{(2)}=\left(t_{1}^{2}-t_{1} t_{2}+t_{2}^{2}\right) B_{(1)} .
$$

Letting $\mathbb{Q}\left\{t_{1}, \ldots, t_{n}\right\}$ be the Laurent polynomial ring, we see that $L^{\prime}(\mathbf{e})$ is the free module $\mathbb{Q}\left\{t_{1}, \ldots, t_{n}\right\} \cdot s$. Identifying $L^{\prime}(\mathbf{e})$ with this Laurent polynomial ring we have:

Theorem 1.4. The image of the map $L(\mathbf{e}) \rightarrow L^{\prime}(\mathbf{e})$ is an ideal in the Laurent polynomial ring. When $\mathbb{k}$ has characteristic 0 or $n=2$, this map is an isomorphism.

The ideal, which is the image of the above map, depends on $\mathbf{e}$ and may depend on the field $\mathbb{k}$; denote it $\mathscr{I}(\mathbb{k} ; \mathbf{e})$. In the case when $\mathbb{k}$ has characteristic $p$ and $n \geq 3$, it is an interesting question to determine this ideal. 
Question 1.5. Is the ideal $\mathscr{I}(\mathbb{k} ; \mathbf{e})$ in the Laurent polynomial ring $\mathbb{Q}\left\{t_{1}, \ldots, t_{n}\right\}$ always nonzero? Is it always equal to the whole ring?

1.4. The associated diagrams when taking total degrees. On the rational rays of pure $\mathbb{Z}$-graded Betti diagrams, it is an open question what integral points come from actual pure resolutions. The following says that in the case of diagrams arising from $\mathbb{Z}^{n}$-graded resolutions of artinian modules over $\mathbb{k}\left[x_{1}, \ldots, x_{n}\right]$, we will not get more than what we get from the equivariant resolution. See however the following remark.

Corollary 1.6. (Char. $\mathbb{k}=0$.) Let $\pi$ be the $\mathbb{Z}^{n}$-graded Betti diagram of a $\mathbb{Z}^{n}$-graded artinian module over $\mathbb{k}\left[x_{1}, \ldots, x_{n}\right]$, whose resolution becomes pure when taking total degrees. Then the associated $\mathbb{Z}$-graded Betti diagram $\bar{\pi}$ is an integer multiple of the associated $\mathbb{Z}$-graded Betti diagram of the equivariant resolution, suitably twisted.

Proof. By Theorems 1.2 and $1.4, \pi$ is a linear combination $\sum_{i} k_{i} \beta_{E}\left(\mathbf{a}_{i}\right)$ where all the $\mathbf{a}_{i}$ have the same total degree, say $a$. Let $P$ be the $(n+1)$-tuple of Laurent polynomials associated to $\pi$, and $s$ the associated $(n+1)$-tuple of Laurent polynomials associated to the equivariant resolution. Then

$$
P=\sum_{i} k_{i} t^{\mathbf{a}_{i}} s,
$$

and we will show that all coefficients here are integers. Considering the first polynomial in the tuple we have $P_{0}=\sum_{i} k_{i} t^{\mathbf{a}_{i}} s_{0}$. Since the highest weight vectors of Schur modules have multiplicity one, the lexicographically largest term of $s_{0}$ has coefficient 1. The coefficient of the highest lexicographic term of $P_{0}$ must then equal $k_{i}$ for some $i$, and so $k_{i}$ is an integer. Then $P_{0}-k_{i} t^{\mathbf{a}_{i}} s_{0}$ has integer coefficients. In this way we may continue and get that all $k_{j}$ are integers. Taking total degress we get

$$
\bar{\pi}=\left(\sum_{i} k_{i}\right) \overline{\beta_{E}}(a) .
$$

Remark 1.7. On the ray generated by the diagram

$$
\tau=\left(\begin{array}{cccc}
1 & 2 & - & - \\
- & - & 2 & 1
\end{array}\right)
$$

the equivariant diagram is $3 \tau$. The above says that it is not possible to realize $2 \tau$ (or $5 \tau$ or $7 \tau$ ) as coming from a $\mathbb{Z}^{3}$-graded diagram over the polynomial ring in three variables. It is however possible to realize $2 \tau$ as coming from a $\mathbb{Z}^{4}$-graded diagram over the polynomial ring $S$ in four variables. Just take a general $\mathbb{Z}^{4}$-graded map :

$$
S_{(0,0,0,0)}^{2} \leftarrow S_{(1,0,0,0)} \oplus S_{(0,1,0,0)} \oplus S_{(0,0,1,0)} \oplus S_{(0,0,0,1)} .
$$

Then $2 \tau$ will be the $\mathbb{Z}$-graded diagram of the resolution of the cokernel. Note however that the cokernel is not artinian.

\section{Schur polynomials}

We describe the greatest common divisor of the Betti polynomials occurring in the equivariant pure resolutions. We do this in Theorem 2.9 and this is the only result of this section that we use later on. 
2.1. Common divisors and group actions. Suppose a group $G$ acts on the polynomial ring $\mathbb{k}\left[t_{1}, \ldots, t_{n}\right]$. A polynomial $p$ is semi-invariant if the groups acts as $g . p=\mu(g) p$ for some character $\mu: G \rightarrow \mathbb{k}$.

Lemma 2.1. Let $p$ and $q$ be semi-invariant polynomials in $\mathbb{k}\left[t_{1}, \ldots, t_{n}\right]$. Then their greatest common divisor is also a semi-invariant.

Proof. If $b$ is the greatest common divisor, then $g . b$ is also a common divisor. Hence $g . b=\mu(g) b$ for some character $\mu$.

We now give two cases where one may actually conclude that if $p$ and $q$ are invariants, their greatest common divisor is also an invariant. Recall the algebra morphism $F_{r}: S \rightarrow S$ of Subsection 1.3.

Lemma 2.2. Let $p$ and $q$ be polynomials in $\mathbb{k}\left[t_{1}, \ldots, t_{n}\right]$ and $b$ their greatest common divisor. For a natural number $r$, the greatest common divisor of $p^{(r)}$ and $q^{(r)}$ is $b^{(r)}$.

Proof. The group $\left(\mathbb{Z}_{r}\right)^{n}$ acts on the polynomial ring, and $p^{(r)}$ and $q^{(r)}$ are invariants. Note that any semi-invariant polynomial for this group has the form $m \cdot c^{(r)}$ for some monomial $m=x_{1}^{a_{1}} \cdots x_{n}^{a_{n}}$ where each $0 \leq a_{i}<r$ and this monomial is uniquely determined by the character. Write $p=m_{1} p_{1}$ where $m_{1}$ is a monomial and $p_{1}$ does not have any monomial as a factor, and similarly $q=n_{1} q_{1}$. Let $m c^{(r)}$ be the greatest common divisor of $p^{(r)}$ and $q^{(r)}$ where $m$ is a monomial and $c^{(r)}$ does not have a monomial factor. Then $m$ divides $m_{1}^{(r)}$ and $n_{1}^{(r)}$, and $m_{1}^{(r)} / m$ and $n_{1}^{(r)} / m$ are semiinvariants with the same character. If this character is non-trivial they will have a common monomial factor. But this is impossible by choice of $c$. Hence $m$ is also an invariant.

Lemma 2.3. The greatest common divisor of two symmetric polynomials in

is also a symmetric polynomial.

$$
\mathbb{k}\left[t_{1}, \ldots, t_{n}\right]
$$

Proof. The symmetric group $S_{n}$ has two characters, the trivial one and the sign of the permutation. If the greatest common divisor $f$ is not symmetric then $\sigma$. $f=(-1)^{\operatorname{sign}(\sigma)} f$. Hence $f$ is divisible by $t_{i}-t_{j}$ for each pair $i<j$ and so by $D=\Pi_{i<j}\left(t_{j}-t_{i}\right)$. But then both $p / D$ and $q / D$ are semi-invariants with the sign character, and so are again divisible by $D$. Thus $f=D^{2} f^{\prime}$ where $f^{\prime}$ is a greatest common divisor of $p / D^{2}$ and $q / D^{2}$. By induction on degree we may assume that $f^{\prime}$ is symmetric.

2.2. Common divisors of Schur polynomials. For a polynomial $f$ in $\mathbb{k}\left[t_{1}, \ldots, t_{n}\right]$ write

$$
f=t_{1}^{N} \bar{f}+\text { lower terms in } t_{1}+t_{1}^{n} \underline{f}
$$

where the last term is the one with the smallest power of $t_{1}$. The polynomials $\bar{f}$ and $\underline{f}$ are in $\mathbb{k}\left[t_{2}, \ldots, t_{n}\right]$. Note that if $f=g h$ then $\bar{f}=\bar{g} \bar{h}$ and $\underline{f}=\underline{g} \underline{h}$. For a partition $\bar{\lambda}=\left(\lambda_{1}, \ldots, \lambda_{n}\right)$ let

$$
\begin{aligned}
\bar{\lambda} & =\left(\lambda_{2}, \ldots, \lambda_{n}\right) \\
\underline{\lambda} & =\left(\lambda_{1}-\lambda_{n}, \ldots, \lambda_{n-1}-\lambda_{n}\right) .
\end{aligned}
$$


By the way Schur polynomials are derived from semi-standard Young tableaux, we see that

$$
\overline{s_{\lambda}}=s_{\bar{\lambda}}, \quad \underline{s_{\lambda}}=\left(t_{2} \cdots t_{n}\right)^{\lambda_{n}} s_{\underline{\lambda}} .
$$

Example 2.4. Let $n=3$ and $\lambda=(4,2,1)$. Then

$$
\begin{aligned}
s_{4,2,1} & =t_{1}^{4} t_{2}^{2} t_{3}+t_{1}^{4} t_{3}^{2} t_{2}+t_{2}^{4} t_{3}^{2} t_{1}+t_{2}^{4} t_{1}^{2} t_{3}+t_{3}^{4} t_{1}^{2} t_{2}+t_{3}^{4} t_{2}^{2} t_{1} \\
& +t_{1}^{3} t_{2}^{3} t_{3}+t_{1}^{3} t_{3}^{3} t_{2}+t_{2}^{3} t_{3}^{3} t_{1}+2 t_{1}^{3} t_{2}^{2} t_{3}^{2}+2 t_{2}^{3} t_{1}^{2} t_{2}^{2}+2 t_{3}^{3} t_{1}^{2} t_{2}^{2} .
\end{aligned}
$$

We get

$$
\begin{aligned}
& \overline{s_{4,2,1}}=t_{2}^{2} t_{3}+t_{3}^{2} t_{2}=s_{2,1} \\
& \underline{s_{4,2,1}}=t_{2}^{4} t_{3}^{2}+t_{3}^{4} t_{2}^{2}+t_{2}^{3} t_{3}^{3}=\left(t_{2} t_{3}\right) s_{3,1}
\end{aligned}
$$

We shall use the notation

$$
\xi_{a}\left(t_{1}, t_{2}\right)=t_{1}^{a-1}+t_{1}^{a-2} t_{2}+\cdots+t_{2}^{a-1}
$$

which factors as $\Pi_{\omega}\left(t_{1}-\omega t_{2}\right)$ where the product is over all $a^{\prime}$ th roots of 1 except 1 itself. Note that this is equal to the Schur polynomial $s_{a-1,0}$. Finally let

$$
\rho=(n-1, n-2, \ldots, 1,0), \quad \rho^{\prime}=(n-2, n-3, \ldots, 1,0) .
$$

Lemma 2.5. Let $f$ be a symmetric polynomial having a non-trivial common factor with $s_{r \rho-\rho}$. Then $s_{p \rho-\rho}$ will divide $f$ for some divisor $p \geq 2$ of $r$.

Proof. We have

$$
s_{r \rho-\rho}=\Pi_{i<j}\left(t_{i}^{r}-t_{j}^{r}\right) / \Pi_{i<j}\left(t_{i}-t_{j}\right)=\Pi_{i<j} \xi_{r}\left(t_{i}, t_{j}\right) .
$$

Suppose, say, $t_{1}-\omega t_{2}$ is a common factor where $\omega \neq 1$ is a primitive $p$ 'th root of unity where $p \geq 2$ divides $r$. Writing

$$
f=\Sigma_{\mathbf{a} \in \mathbb{Z}^{n-2}} t_{3}^{a_{3}} \cdots t_{n}^{a_{n}} p_{\mathbf{a}}\left(t_{1}, t_{2}\right)
$$

where the $p_{\mathbf{a}}\left(t_{1}, t_{2}\right)$ are symmetric polynomials over $\mathbb{Z}$, we see that $t_{1}-\omega t_{2}$ is a factor of each $p_{\mathbf{a}}\left(t_{1}, t_{2}\right)$. Hence $\xi_{p}\left(t_{1}, t_{2}\right)$ is a factor of $f$. Since $f$ is symmetric, all $\xi_{p}\left(t_{i}, t_{j}\right)$ must divide $f$ and so $s_{p \rho-\rho}$ will divide $f$.

Lemma 2.6. If $r$ is a common divisor of $\lambda_{1}, \ldots, \lambda_{n}$, write $\lambda=r \cdot \lambda^{\prime}$ for a partition $\lambda^{\prime}$. Then

$$
s_{\lambda-\rho}=s_{\lambda^{\prime}-\rho}^{(r)} \cdot s_{r \rho-\rho} .
$$

Proof. The following short argument was brought to our attention by J.Weyman and S.Sam.

$$
s_{\lambda-\rho}=\frac{\left|t_{i}^{\lambda_{j}}\right|}{\left|t_{i}^{n-j}\right|}=\frac{\left|t_{i}^{\lambda_{j}}\right|}{\left|t_{i}^{r(n-j)}\right|} \cdot \frac{\left|t_{i}^{r(n-j)}\right|}{\left|t_{i}^{n-j}\right|}=s_{\lambda^{\prime}-\rho}^{(r)} \cdot s_{r \rho-\rho} .
$$

Lemma 2.7. For any $\lambda$ and $r$, the polynomials $s_{\lambda}^{(r)}$ and $s_{r \rho-\rho}$ are relatively prime. 
Proof. If $n=2$ then

$$
s_{\lambda_{1}, \lambda_{2}}^{(r)}=\left(t_{1} t_{2}\right)^{\lambda_{2} r} \cdot s_{\lambda_{1}-\lambda_{2}, 0}^{(r)} .
$$

So we must show that for any $a$, the polynomials $s_{a-1,0}^{(r)}$ and $s_{r-1,0}$ are relatively prime. These polynomials are

$$
\frac{t_{1}^{a r}-t_{2}^{a r}}{t_{1}^{r}-t_{2}^{r}} \text { and } \frac{t_{1}^{r}-t_{2}^{r}}{t_{1}-t_{2}}
$$

Since $t_{1}^{a r}-t_{2}^{a r}$ does not have any multiple factors, these are relatively prime.

Let now $n \geq 3$. If the polynomials in the statement have a greatest common divisor $f$, then $\bar{f}$ is a common divisor of of $\overline{s_{\lambda}^{(r)}}={\overline{s_{\lambda}}}^{(r)}=s_{\bar{\lambda}}^{(r)}$ and $\overline{s_{r \rho-\rho}}=s_{r \rho^{\prime}-\rho^{\prime}}$. By induction the greatest common divisor of these is 1 . If $f$ is not 1 it has by Lemma 2.5 a factor of the form $s_{p \rho-\rho}$ for some $p \geq 2$ dividing $r$. But then $s_{p \rho^{\prime}-\rho^{\prime}}$ would be a factor of $\bar{f}=1$.

Lemma 2.8. Suppose $\lambda-\rho$ is non-negative and $r$ is relatively prime to at least one $\lambda_{i}-\lambda_{i+1}$ where $1 \leq i \leq n-1$. Then $s_{\lambda-\rho}$ and $s_{r \rho-\rho}$ are relatively prime.

Proof. If $n=2$, then

$$
s_{\lambda_{1}-1, \lambda_{2}}=\left(t_{1} t_{2}\right)^{\lambda_{2}} s_{\lambda_{1}-\lambda_{2}-1,0} .
$$

This is relatively prime to $s_{r-1,0}$, since $\xi_{p}\left(t_{1}, t_{2}\right)$ and $\xi_{q}\left(t_{1}, t_{2}\right)$ are relatively prime when $p$ and $q$ are.

Let $n \geq 3$. If $s_{\lambda-\rho}$ and $s_{r \rho-\rho}$ have a non-trivial common factor they have a common factor $s_{p \rho-\rho}$ where $p \geq 2$ divides $r$. Suppose that $p$ is relatively prime to $\lambda_{1}-\lambda_{2}$. Then $s_{p \rho-\rho}$, which is $\left(t_{2} \cdots t_{n}\right)^{p-1} \cdot s_{p \rho^{\prime}-\rho^{\prime}}$, is a common factor of $s_{\lambda-\rho}$ and $s_{r \rho-\rho}$ which are respectively

$$
\left(t_{2} \cdots t_{n}\right)^{\lambda_{n}-1} \cdot s_{\underline{\lambda}-\rho^{\prime}} \text { and }\left(t_{2} \cdots t_{n}\right)^{r-1} \cdot s_{r \rho^{\prime}-\rho^{\prime}} .
$$

Then $s_{p \rho^{\prime}-\rho^{\prime}}$ would have to be a common factor of $s_{\underline{\lambda}-\rho^{\prime}}$ and $s_{r \rho^{\prime}-\rho^{\prime}}$ which by induction is not possible.

Now assume that $p$ is relatively prime to $\lambda_{i}-\lambda_{i+1}$ for some $2 \leq i \leq n-1$. Then $\overline{s_{p \rho-\rho}}=s_{p \rho^{\prime}-\rho^{\prime}}$ is a common factor of $s_{\bar{\lambda}-\rho^{\prime}}$ and $s_{r \rho^{\prime}-\rho^{\prime}}$. But by induction these two latter polynomials are relatively prime, so again we get a contradiction.

Now we are ready to prove the main result of this subsection.

Theorem 2.9. Let $r$ be the greatest common divisor of $e_{1}, \ldots, e_{n}$. Then $s_{r \rho-\rho}$ is the greatest common divisor of

$$
s_{\alpha(\mathbf{e}, 0)}, s_{\alpha(\mathbf{e}, 1)}, \ldots, s_{\alpha(\mathbf{e}, n)} .
$$

Letting $e_{i}=r e_{i}^{\prime}$ we have

$$
s_{\alpha(\mathbf{e}, i)}=s_{r \rho-\rho} \cdot s_{\alpha\left(\mathbf{e}^{\prime}, i\right)}^{(r)} .
$$

Proof. 1. The last equation is by Lemma 2.6. To show the statement, it is by Lemma 2.2 enough to show that if the greatest common divisor $r=1$, then the greatest common divisor of the $s_{\alpha(\mathbf{e}, i)}$ 's is 1 . We do this by induction on $n$. When $n=2$, the Schur polynomials are

$$
s_{\left(e_{2}-1,0\right)}, \quad s_{\left(e_{1}+e_{2}-1,0\right)}, \quad s_{\left(e_{1}+e_{2}-1, e_{2}\right)}=\left(t_{1} t_{2}\right)^{e_{2}} s_{\left(e_{1}-1,0\right)} .
$$


The first polynomial is $\xi_{e_{2}}$ and the last polynomial is $\xi_{e_{1}}$, and these are relatively prime when $e_{1}$ and $e_{2}$ are.

2. Suppose then $n \geq 3$. Let $a$ be the greatest common divisor of $e_{1}, \ldots, e_{n-1}$ and $b$ the greatest common divisor of $e_{2}, \ldots, e_{n}$. Then $a$ and $b$ are relatively prime. Let $f$ be the greatest common divisor of the $s_{\alpha(\mathbf{e}, i)}$ for $i=0, \ldots, n$. It is symmetric by Lemma 2.3. Also $f$ does not have any variables as a factor. Otherwise it would be divisible by $t_{1} t_{2} \cdots t_{n}$ but this does not go together with $f$ dividing $s_{\alpha(\mathbf{e}, 0)}$.

By Lemma 2.6 note that

$$
s_{\alpha(\mathbf{e}, 0)}=s_{b \rho-\rho} \cdot s_{\alpha\left(\mathbf{e}^{\prime}, 0\right)}^{(b)}
$$

where $\mathbf{e}^{\prime}=\left(*, e_{2} / b, \ldots, e_{n} / b\right)$ (by Lemma 1.1 the last factor above does not depend on the first coordinate of $\left.\mathbf{e}^{\prime}\right)$. Also

$$
s_{\alpha(\mathbf{e}, n)}=s_{a \rho-\rho} \cdot\left(t_{1} \cdots t_{n}\right)^{e_{n}} \cdot s_{\alpha\left(\mathbf{e}^{\prime \prime}, 0\right)}^{(a)}
$$

where $\mathbf{e}^{\prime \prime}=\left(*, e_{1} / a, \ldots, e_{n-1} / a\right)$ (again by Lemma 1.1 the last factor above does not depend on the first coordinate of $\left.\mathbf{e}^{\prime \prime}\right)$. Now $f$ will be relatively prime to $s_{b \rho-\rho}$ and $s_{a \rho-\rho}$. This is so since $s_{b \rho-\rho}$ is relatively prime to $s_{\alpha(\mathbf{e}, n)}$ by Lemma 2.8 because $\alpha(\mathbf{e}, n)_{1}-\alpha(\mathbf{e}, n)_{2}+1=e_{1}$, and since $s_{a \rho-\rho}$ is relatively prime to $s_{\alpha(\mathbf{e}, 0)}$ by Lemma 2.8 because $\alpha(\mathbf{e}, 0)_{n-1}-\alpha(\mathbf{e}, n)_{n}+1=e_{n}$. Hence we may conclude that $f$ is a common factor of $s_{\alpha\left(\mathbf{e}^{\prime}, 0\right)}^{(b)}$ and $s_{\alpha\left(\mathbf{e}^{\prime \prime}, 0\right)}^{(a)}$.

3. Now we consider $\bar{f}$. It divides

$$
\overline{s_{\alpha\left(\mathbf{e}^{\prime}, 0\right)}^{(b)}}=\overline{s_{\alpha\left(\mathbf{e}^{\prime}, 0\right)}}(b)=s \frac{(b)}{\alpha\left(\mathbf{e}^{\prime}, 0\right)}=s_{\alpha\left(\overline{\mathbf{e}}^{\prime}, 0\right)}^{(b)}
$$

where $\overline{\mathbf{e}}^{\prime}=\left(*, e_{3} / b, \ldots, e_{n} / b\right)$. But it also divides the $\overline{s_{\alpha(\mathbf{e}, i)}}$ and for $i \geq 1$ these are by (5) and (7) equal to

$$
s_{\alpha(\overline{\mathbf{e}}, i)}, \quad i \geq 1
$$

where $\overline{\mathbf{e}}=\left(e_{2}, \ldots, e_{n}\right)$. By induction the greatest common divisor of these polynomials is $s_{b \rho^{\prime}-\rho^{\prime}}$. We may then by Lemma 2.7 conclude that $\bar{f}=1$. Since $f$ is symmetric we have

$$
f=t_{1}^{m}+\text { lower terms in } t_{1}+\underline{f}
$$

where $f$ does not have any variable as a factor.

4. Now consider $\underline{f}$. We know that $f$ divides $s_{\alpha\left(\mathbf{e}^{\prime \prime}, 0\right)}^{(a)}$. Note that

$$
\underline{s_{\alpha\left(\mathbf{e}^{\prime \prime}, 0\right)}}=\left(t_{2} \cdots t_{n}\right)^{\mathbf{e}_{n}^{\prime \prime}-1} \cdot s_{\alpha\left(\underline{\mathbf{e}}^{\prime \prime}, 0\right)}
$$

where $\underline{\mathbf{e}}^{\prime \prime}=\left(*, e_{1} / a, \ldots, e_{n-2} / a\right)$. Hence $\underline{f}$ divides $s_{\alpha\left(\underline{\underline{e}}^{\prime \prime}, 0\right)}^{(a)}$.

But $\underline{f}$ also divides $s_{\alpha(\mathbf{e}, i)}$ for $i=0, \ldots, n-1$ which is

$$
\left(t_{2} \cdots t_{n}\right)^{e_{n}-1} \cdot s_{\alpha(\hat{\mathbf{e}}, i)}
$$

where $\hat{\mathbf{e}}=\left(e_{1}, \ldots, e_{n-1}\right)$. Hence $\underline{f}$ is a common factor of the $s_{\alpha(\hat{\mathbf{e}}, i)}$. By induction their greatest common divisor is $\bar{s}_{a \rho^{\prime}-\rho^{\prime}}$. By Lemma 2.7 we may now conclude that $\underline{f}=1$. Since $f$ is symmetric we may further conclude that $f=1$. 


\section{The linear space of diagrams fulfilling the Herzog-Kühl equation}

The theorem below provides a nice structural description of the $\mathbb{Q}$-vector space $L^{\prime}(\mathbf{e})$ of diagrams fulfilling the HK-equations (4). Recall again that $L^{\prime}(\mathbf{e})$ does not depend on the field $\mathbb{k}$. Using this theorem and Theorem 2.9, which gives the common factor of the Schur polynomials, the proofs of our main Theorems 1.2 and 1.4 are rather immediate.

Theorem 3.1. Let $\mathbf{e}=\left(e_{1}, \ldots, e_{n}\right)$ be a vector of positive integers.

a. There is an $(n+1)$-tuple $\left(A_{0}, \ldots, A_{n}\right)$ of homogeneous Laurent polynomials in $n$ variables, such that $L^{\prime}(\mathbf{e})$ has a basis consisting of all $t^{\mathbf{a}}\left(A_{0}, \ldots, A_{n}\right)$ where $\mathbf{a}$ varies in $\mathbb{Z}^{n}$.

$b$. The $A_{i}$ 's have no common factors except for units (which are products of nonzero constants and Laurent monomials $t^{\mathbf{a}}$ ), and are uniquely determined up to common multiplication by a unit.

We prove this towards the end of this section. As a consequence of the above theorem we can prove our main theorems.

Proof of Theorem 1.2. Let $\mathbf{e}=r \cdot \mathbf{e}^{\prime}$. The associated $(n+1)$-tuple of Betti polynomials to the complex $E\left(\mathbf{e}^{\prime}\right)^{(r)}$ is ( when $\mathbb{k}$ has characteristic 0 )

$$
s=\left(s_{\alpha\left(\mathbf{e}^{\prime}, 0\right)}^{(r)}, \ldots, s_{\alpha\left(\mathbf{e}^{\prime}, n\right)}^{(r)}\right) .
$$

This will be a multiple of $\left(A_{0}, \ldots, A_{n}\right)$ in Theorem 3.1 above. By Theorem 2.9 the greatest common divisor of these Schur polynomials is 1 . Hence we can take them to be equal to the $A_{i}$ 's. So if $B$ is in $L^{\prime}(\mathbf{e})$, then $B=p s$ for some Laurent polynomial $p$. And if $B$ is integral, it follows by the same argument as in Corollary 1.6, that $p$ must have integer coefficients, proving the first part of Theorem 1.2.

To prove the second part of Theorem 1.2, note that if $A^{\prime}$ is another element in $L^{\prime}(\mathbf{e})$ with the property of $A$, it must be $\gamma t^{\mathbf{a}} A$ for some rational number $\gamma$ and $\mathbf{a}$ in $\mathbb{Z}^{n}$. But when the coefficients of the polynomials in $A^{\prime}$ are integers then $\gamma$ must be an integer, since the highest weight of the Schur modules $S_{\alpha\left(\mathbf{e}^{\prime}, i\right)}$ always occurs with multiplicity one. And if $\gamma t^{\mathbf{a}} A$ is part of a lattice basis, only the values $\gamma= \pm 1$ can occur.

Proof of Theorem 1.4. If $E$ is a resolution with Betti polynomials $B$, then $E(\mathbf{a})$ has Betti polynomials $t^{\text {a }} B$. Hence the image of $L(\mathbf{e}) \rightarrow L^{\prime}(\mathbf{e})$, where the latter is $\mathbb{Q}\left\{t_{1}, \ldots, t_{n}\right\} \cdot s$, identifies as an ideal in the Laurent polynomial ring.

If $\mathbb{k}$ has characteristic 0 , the generator $s$ is in the image so the map is an isomorphism. If $n=2$ it is shown in [7], Proposition 3.1, that there exists resolutions with Betti polynomials $s$, regardless of $\mathbb{k}$.

\subsection{Properties of tuples fulfilling the HK-equations.}

Proposition 3.2. Let $B=\left(B_{0}, \ldots, B_{n}\right)$ be an homogeneous $(n+1)$-tuple in $L^{\prime}(\mathbf{e})$.

a. If $B_{0}=0$ then $B=\mathbf{0}$.

Assume $B_{0}$ is nonzero. Let $B_{0}=t_{1}^{b_{1}} B_{0}^{*}+$ lower terms in $t_{1}$, where $B_{0}^{\prime}$ is a Laurent polynomial in $t_{2}, \ldots, t_{n}$.

b. Then $B_{1}=t_{1}^{b_{1}+e_{1}} B_{0}^{*}+$ lower terms in $t_{1}$. 
c. Each $B_{i}$ is nonzero and the highest power of $t_{1}$ occurring in $B_{i}$ for $i \geq 1$ is $t_{1}^{b_{1}+e_{1}}$.

Proof. Note that the statement holds when $n=1$. We shall then work further using induction. Let $u$ be the smallest index such that $B_{u} \neq 0$. Suppose $B_{u}=t_{1}^{p_{1}} B_{u}^{*}+$ lower terms in $t_{1}$. Let $t_{2}^{p_{2}} \cdots t_{n}^{p_{n}}$ be a monomial in $B_{u}^{*}$. Since the total degree of $B_{u}$ is fixed equal to $d_{u}$, then in $B_{u}$ this term occurs only with $t_{1}^{p_{1}}$ as the power of $t_{1}$. So let $B_{u}=c_{\mathbf{p}} t_{1}^{p_{1}} \cdots t_{n}^{p_{n}}+$ other terms, where the coefficient $c_{\mathbf{p}}$ is nonzero.

The Herzog-Kühl equations give, by the projection omitting the first coordinate, that some $B_{v}$, where $v>u$, will contain the monomial $t_{1}^{p_{1}+d_{v}-d_{u}} t_{2}^{p_{2}} \cdots t_{n}^{p_{n}}$ (denoting the degree of $B_{i}$ by $d_{i}$ ).

Let $A_{1}$ be the highest power of $t_{1}$ occurring in any $B_{i}$. Write $B=t_{1}^{A_{1}} B^{\prime}+$ lower terms in $t_{1}$, where $B^{\prime}$ is a nonzero homogeneous $(n+1)$-tuple in the variables $t_{2}, \ldots, t_{n}$. By what we have shown $A_{1} \geq p_{1}+d_{v}-d_{u}$. This gives $A_{1}>p_{1}$ and the smallest $u^{\prime}$ for which $B_{u^{\prime}}^{\prime}$ is nonzero must be $>u$. By omitting $B_{0}^{\prime}$ (which is zero) we may consider $B^{\prime}$ as an $n$-tuple in $t_{2}, \ldots, t_{n}$. Also we see that it will satisfy the Herzog-Kühl equations for $n$-tuples, by looking at the equations satisfied by $B$ when we always keep the first coordinate equal to $A_{1}$. By induction on $n$ we get that $B_{1}^{\prime}$ is nonzero and so the index $u$ must be 0 . This proves a. and shows that $b_{1}=p_{1}$. Also, by induction from c. we get that each $B_{i}^{\prime}$ is nonzero, for $i \geq 1$. Hence we get $B_{i}$ nonzero for $i \geq 1$, and we also have shown $B_{0}$ nonzero, proving the first part of $\mathrm{c}$.

Let $t_{2}^{q_{2}} \cdots t_{n}^{q_{n}}$ be a term occurring in $B_{1}^{\prime}$. The Herzog-Kühl equations with projection omitting the first coordinate, gives that $t_{1}^{A_{1}-e_{1}} t_{2}^{q_{2}} \cdots t_{n}^{q_{n}}$ occurs as a term in $B_{0}$. Hence $A_{1}-e_{1} \leq b_{1}$. Since we also have $A_{1} \geq b_{1}+e_{1}$ we get $A_{1}=b_{1}+e_{1}$. Since the $B_{i}^{\prime}$ are nonzero this also proves the second part of c.

Now if $c_{\mathbf{p}} t_{1}^{p_{1}} \cdots t_{n}^{p_{n}}$ is a term in $B_{0}$ with $p_{1}=b_{1}$, it follows by the HK-equations, by the projection omitting the first coordinate, that $t_{1}^{p_{1}+\sum_{i=1}^{v} e_{i}} t_{2}^{p_{2}} \cdots t_{n}^{p_{n}}$ occurs as a term in $B_{v}$ for some $v$. Since $A_{1}=b_{1}+e_{1}$ this only happens for $v=1$ and with coefficient $c_{\mathbf{p}}$, thus proving $\mathrm{b}$.

Lemma 3.3. Let $p$ be a homogeneous Laurent polynomial and $B$ a homogeneous $(n+1)$-tuple. Then $p \cdot B$ fulfils the HK-equations if and only if $B$ fulfils these equations.

Proof. The if direction is clear. So suppose $p \cdot B$ fulfils the HK-equations but $B$ does not. So for some $n-1$-tuple $\hat{\mathbf{a}}$ the equation (4) is not fulfilled. By re-indexing we may assume that $\hat{\mathbf{a}}$ are the first coordinates in a, i.e. $k=n$ in (4).

Also suppose $\hat{\mathbf{a}}$ is the lexicographic largest $(n-1)$-tuple such that the HK-equations for $B$ do not hold. Write $p=t_{1}^{\lambda_{1}} t_{2}^{\lambda_{2}} \cdots t_{n}^{\lambda_{n}}+$ lower terms for the lex order. Then we see that for the $(n-1)$-tuple

$$
\left(\lambda_{1}, \lambda_{2}, \ldots, \lambda_{n-1}\right)+\left(\hat{a}_{1}, \hat{a}_{2}, \ldots, \hat{a}_{n-1}\right),
$$

the HK-equations for $p \cdot B$ where we keep the first $n-1$ coordinates fixed equal to the $(n-1)$-tuple above, does not hold.

Corollary 3.4. If $p$ is any Laurent polynomial, and $B$ is any $(n+1)$-tuple of Laurent polynomials, then $B$ is in $L^{\prime}(\mathbf{e})$ if and only if $p \cdot B$ is in $L^{\prime}(\mathbf{e})$. In particular $L^{\prime}(\mathbf{e})$ is a submodule of $L^{n+1}$.

Proof. This is because $L^{\prime}(\mathbf{e})$ is a graded vector space. 
Given a Laurent polynomial $B_{0}$, let $t_{1}^{c_{1}} \cdots t_{n}^{c_{n}}$ be the lexicographic largest term in $B_{0}$. For each $i=1, \ldots, n$ let $b_{i}$ be the smallest integer such that

$$
t_{1}^{c_{1}} \cdots t_{i-1}^{c_{i-1}} t_{i}^{b_{i}} t_{i+1}^{d_{i+1}} \cdots t_{n}^{d_{n}}
$$

is in $B_{0}$ for some choice of $d_{i+1}, \ldots, d_{n}$. We define the valuation of $B_{0}$ to be

$$
\left(c_{1}-b_{1}, c_{2}-b_{2}, \ldots, c_{n}-b_{n}\right) .
$$

Example 3.5. The valuation of the Schur polynomial $s_{4,2,1}$ of Example 2.4 is $(3,1,0)$.

The valuation is in $\mathbb{N}^{n}$ (note however that $c_{n}-b_{n}$ is always zero). We now order $\mathbb{N}^{n}$ lexicographically with $0<1<2<\cdots$. We may note that $\mathbb{N}^{n}$ with this ordering is a well-ordered set, i.e. each subset has a smallest element. When $B$ is an $(n+1)$ tuple of Laurent polynomials with $B_{0}$ nonzero, we define the valuation of $B$ to be the valuation of $B_{0}$.

3.2. The abstract situation. To give a more transparent argument we will now abstract our situation. Let $L$ be an integral domain, and $M$ a submodule of $L^{n+1}$. Suppose we have a map $v: M \backslash\{0\} \rightarrow T$, which we call a valuation, to a well ordered set $T$, subject to the following requirements.

1. If $p$ is in $L$ and $b$ in $M$, then $v(p b) \geq v(b)$.

2. If $b$ is in $L^{n+1}$ and $p$ in $L$, then $p b$ is in $M$ if and only if $b$ is in $M$.

3. Let $a$ and $b$ be in $M$. Then there exists nonzero $p$ and $q$ in $L$ such that $p a-q b$ is either zero or has valuation $<\max \{v(a), v(b)\}$.

Note that if $L$ is the Laurent polynomial ring, $M$ is $L^{\prime}(\mathbf{e})$, and $T=\mathbb{N}^{n}$, by letting $v(B)$ be the valuation as defined in the end of the preceding subsection, it fulfils 1 . and 2. Note that $v$ is welldefined since $B_{0}$ is nonzero if $B$ is nonzero.

We shall later show that it fulfils 3 . But let us assume that we have a valuation as above. We then get a stronger version of 3 .

Lemma 3.6. Given a valuation $v$ as above, and let $a$ and $b$ be nonzero in $M$. Then there are nonzero $p$ and $q$ in $L$ such that $p a-q b$ is either zero or has valuation $<\min \{v(a), v(b)\}$.

Proof. Suppose $v(a) \leq v(b)$. We can then find nonzero $p_{1}$ and $q_{1}$ such that $b^{\prime}=$ $q_{1} b-p_{1} a$ is zero or has valuation $<v(b)$. If $b^{\prime}$ is nonzero with valuation $\geq a$, we may continue and find nonzero $p_{2}$ and $q_{2}$ such that

$$
b^{\prime \prime}=q_{2} b^{\prime}-p_{2} a=q_{2} q_{1} b-\left(p_{1} q_{2}+p_{2}\right) a
$$

is either zero or has valuation $<v\left(b^{\prime}\right)$. In this way we may continue. If the process does not stop we have an infinite strictly decreasing chain of valuations, contrary to $T$ being well-ordered. Hence for some $n$ we obtain

$$
\begin{aligned}
b^{(n)} & =q_{n} \cdot b^{(n-1)}-p_{n} \cdot a \\
& =q_{n} q_{n-1} \cdots q_{1} \cdot b-p_{n}^{\prime} a
\end{aligned}
$$

(for some $p_{n}^{\prime}$ ) which has valuation $<v(a)$, or is zero. Note that $q_{n} q_{n-1} \cdots q_{1}$ is nonzero. By 1. we must also have $p_{n}^{\prime}$ nonzero.

We are now ready to prove our structure result for valuations fulfilling requirements 1., 2 . and 3 . 
Proposition 3.7. Suppose $L$ is a unique factorisation domain, and $M$ is a submodule of $L^{n+1}$ with a valuation fulfilling 1., 2., and 3. Then there is an a in $M$ such that $M$ is the submodule of $L^{n+1}$ generated by a. Any a in $M$ such that the greatest common divisor of its components $a_{1}, \ldots, a_{n+1}$ is 1 , is such a generator.

Proof. Given the first statement, the second is clear. Let $a$ be a nonzero element of $M$ with the smallest possible valuation. Then we may write $a=p a^{\prime}$ for some polynomial $p$ where the components of $a^{\prime}$ has 1 as their greatest common divisor. By axiom 1. $v\left(a^{\prime}\right) \leq v(a)$ and so we may assume that $a$ is $a^{\prime}$. Now choose any nonzero $b$ in $M$. Then there are nonzero $p$ and $q$ such that $q b-p a$ is either zero or has valuation $<v(a)$. The latter is not so by assumption, so $q b=p a$. We factor out any common factors of $p$ and $q$. But then by unique factorisation and construction of $a$ we must have $q$ a unit.

We will now show that the property 3 . holds in our case when $L$ is the Laurent polynomial ring in $n$ variables and $M$ is the submodule $L^{\prime}(\mathbf{e})$.

Proposition 3.8. Let $A$ and $B$ be $(n+1)$-tuples in $L^{\prime}(\mathbf{e})$ and $v$ the valuation defined at the end of Subsection 3.1. Then there are nonzero Laurent polynomials $p$ and $q$ such that $p A-q B$ is zero or has valuation $<\max \{v(A), v(B)\}$.

Proof. If $n=1$ then $A=\left(\alpha t^{a}, \alpha t^{a+e_{1}}\right)$ and $B=\left(\beta t^{b}, \beta t^{b+e_{1}}\right)$ so this clearly holds.

Suppose $n \geq 2$. By adjusting $A$ and $B$ by units, actually Laurent monomials $t^{\mathbf{c}}$, we may assume that the leading terms of the first polynomials in $A$ and $B$ for the lex order are their valuations. (In Example 3.5 this amounts to replacing $s_{4,2,1}$ by $t_{1}^{-1} t_{2}^{-1} t_{3}^{-1} s_{4,2,1}$.) Let

$$
\begin{aligned}
& A=t_{1}^{a_{1}} A^{\prime}+\text { lower terms in } t_{1} \\
& B=t_{1}^{b_{1}} B^{\prime}+\text { lower terms in } t_{1},
\end{aligned}
$$

and assume $b_{1} \geq a_{1}$. Then the valuation of $A^{\prime}$ is the projection $\pi_{1}(v(A))$ and the valuation of $B^{\prime}$ is $\pi_{1}(v(B))$. By induction on $n$ and Lemma 3.6 we may find nonzero $p$ and $q$ in variables $t_{2}, \ldots, t_{n}$ such that $q B^{\prime}-p A^{\prime}$ is zero or has valuation less than that of both $\pi_{1}(v(A))$ and $\pi_{1}(v(B))$. But then $q B-t_{1}^{b_{1}-a_{1}} p A$ will have valuation less than the maximum of $v(A)$ and $v(B)$.

We may now finish off.

Proof of Theorem 3.1. Parts a. and b. follow from Proposition 3.7 by letting $L$ be the Laurent polynomial ring in the variables $t_{1}, \ldots, t_{n}$, and $v$ the valuation defined at the end of Subsection 3.1. This is a valuation by Proposition 3.8.

\section{Acknowledgements}

I thank M.Boij and J.Weyman for discussions concerning this paper. 


\section{References}

[1] M. Boij and J. Soderberg, Graded Betti numbers of Cohen-Macaulay modules and the multiplicity conjecture, Journal of the London Mathematical Society 78 (2008), no. 1, 78-101.

[2] D. Eisenbud, D. Erman, and F. Schreyer, Beyond Numerics: The Existence of Pure Filtrations, Arxiv preprint arXiv:1001.0585 (2010)

[3] D. Eisenbud, G. Fløystad, and J. Weyman, The existence of pure free resolutions, ArXiv preprint arXiv:0709.1529 (2007) To appear in Annales de l'institut Fourier.

[4] D. Eisenbud and F. Schreyer, Betti numbers of graded modules and cohomology of vector bundles, Journal of the American Mathematical Society 22 (2009), no. 3, 859-888.

[5] Erman, D., The semigroup of Betti diagrams, Algebra and Number Theory 3 (2009), no. 3, 341-365.

[6] W. Fulton and J. Harris, Representation theory: A first course, Springer (1991).

[7] B. M. and G. Fløystad, The positive cone of Betti diagrams of bigraded artinian modules of codimension two, ArXiv preprint arXiv:1001.3238 (2010)

[8] S. Sam and J. Weyman, Pieri resolutions for classical groups, Journal of Algebra (2010)

Matematisk Institutt, University of Bergen, Johs. Brunsgt. 12, 5008 Bergen, Norway E-mail address: gunnar@mi.uib.no 